\title{
Cross-Layer Design of Dynamic Bandwidth Allocation in DVB-RCS
}

\author{
Antoni Morell, Student Member, IEEE, Gonzalo Seco-Granados, Member, IEEE, and \\ María Ángeles Vázquez-Castro, Member, IEEE
}

\begin{abstract}
In this paper, a novel cross-layer framework for optimizing the dynamic bandwidth allocation (DBA) of a digital video broadcast (DVB)-return channel satellite (RCS) system using adaptive coding is proposed. The design of the medium access control (MAC) methods taking into account the adaptive physical layer and higher layers' quality of service $(\mathrm{QoS})$ requirements is cast as an optimization problem by using the network utility maximization (NUM) framework applied within the satellite subnetwork. Hierarchical and global solving procedures fully compliant with the DVB-RCS standard are proposed. They do not only provide minimum bandwidth guarantees but also maximize fairness. Further, they allow a joint optimization of the time slot size and overall system efficiency while minimizing signalling overhead. A reduced computational complexity algorithm to solve the DBA problem is presented. In practical terms, it increases the number of connections with absolute and relative $Q 0 S$ requirements the system can manage and facilitates the interoperability of the satellite network within an Internet protocol (IP) environment.
\end{abstract}

Index Terms-Cross layer, digital video broadcast (DVB)-return channel satellite (RCS), dynamic bandwidth allocation (DBA), optimization algorithms, satellite communications.

\section{INTRODUCTION}

A S INTERNET traffic continues to grow, satellite systems need to move from fixed bandwidth services (such as voice and video broadcasting) to dynamic bandwidth Internet Protocol (IP)-based services. Moreover, recent years have seen increasing interest in delivering IP-based multimedia applications requiring broadband support [1]. The digital video broadcasting-satellite (DVB-S) [2] is a widely accepted standard in the forward link of broadband satellite communications. The second generation (DVB-S2) [3] includes the transmission of multimedia contents and a variety of unicast and multicast services. In that context, a satellite terminal is intended to offer a huge package of services to the end-user.

These wide system possibilities of DVB-S2 need interactivity and thus, a return link over the satellite is mandatory (a terrestrial return link would limit the expansion of DVB-S2 to certain areas). The current counterpart of DVB-S2 for the return link is the DVB-return channel satellite (RCS) standard [4], [5]. This paper focuses on unicast services for which adaptive coding and

Manuscript received November 12, 2007; revised November 29, 2007. This work was supported in part by MEC projects ESP2005-03403 and ESP200626372-E and by ESA Project AO/1-4761/05/NL/AD.

The authors are with the Department of Telecommunications and System Engineering, Universitat Autónoma de Barcelona, Barcelona 08193, Spain (e-mail: antoni.morell@uab.cat; gonzalo.seco@uab.cat; angeles.vazquez@uab.cat).

Color versions of one or more of the figures in this paper are available online at http://ieeexplore.ieee.org.

Digital Object Identifier 10.1109/JSYST.2007.914812 modulation (ACM) has been made mandatory in the DVB-S2 standard while adaptive coding is allowed in the DVB-RCS standard. To our knowledge, while the adaptive nature of the forward link is attracting increasing interest [6], [7], the adaptive nature of the DVB-RCS still requires research effort. Previous works include [8] and [9].

A novel cross-layer framework is proposed for optimizing the dynamic bandwidth allocation (DBA) for unicast multimedia services in the return link [10]. Generally speaking, two main questions must be focused in the design phase of good DBA procedures in the DVB-RCS context:

1) How much structure do we impose to the multiple access scheme?

2) Within a given structure, how are resources optimally distributed?

Note the tradeoff between computational complexity and optimality, i.e., in a highly structured multiple-access scheme, few degrees of freedom are left to resource optimization, whereas no structure may result into computationally unaffordable allocation approaches. In this paper, a balanced solution is found. Moreover, bandwidth allocation considers physical (PHY) layer information (the spectral efficiency of each DVB-RCS terminal) and also the required quality of service (QoS) for each traffic type at the upper layers. The resulting cross-layer DBA problem is cast in the framework of network utility maximization (NUM) [11], [12], which in turn, maximizes fairness among users. The main goal is to merge the satellite subnetwork in a QoS-based IP environment. Ideally, to make the satellite link transparent to the network.

This paper is organized as follows. In Section II, we provide an overview of general issues concerning DBA in DVB-RCS. Section III introduces the proposed framework for DBA, i.e., our choice for an standard-compliant structure for the multipleaccess scheme. Section IV is devoted to procedures and algorithms for DBA optimization, whereas Section V includes further system optimization as well as the structure introduced in Section III is tuned up. Finally, we present numerical results in Section VI and the conclusion in Section VII.

\section{Dynamic Bandwidth Allocation In the DVB-RCS}

We consider a transparent satellite network as depicted in Fig. 1. In this paper, we analyze the process carried out by the network control center (NCC), which is the entity that collects demands [13]-[15], runs the DBA algorithm and sends the resulting allocation out to the return channel satellite terminals (RCSTs).

Bandwidth allocation techniques allowed by the DVB-RCS standard belong to the class called resource reservation on-demand [16] or demand-assignment multiple access (DAMA). 


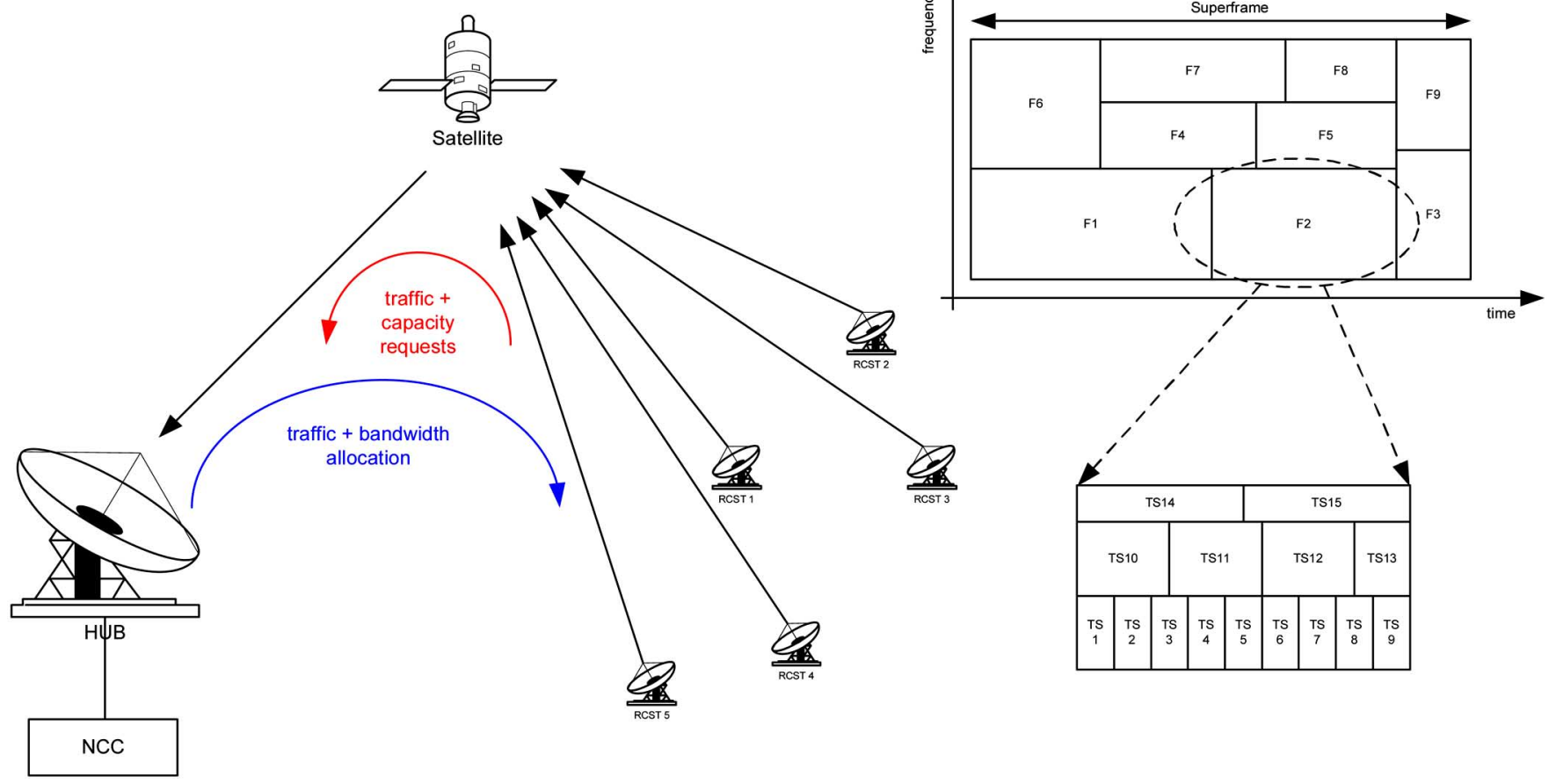

Fig. 1. System overview.

Bandwidth reservation messages are sent from the RCSTs to the NCC and a centralized scheduler applies the DBA algorithms. The bandwidth allocation is sent back to the RCSTs at least one round trip time (RTT) after the request was sent. RCSTs capacity requests can be emitted every RTT; however, not all the stations will request bandwidth continuously. It is important to note here the challenging allocation problem: while the IP traffic is inherently connectionless, DAMA algorithms actually set up a connection over the DVB-RCS air interface [17], which is multi-frequency-time division multiple access (MF-TDMA). The MF-TDMA can be almost freely configured according to the standard. The highest level of division is constituted by the superframe (SF) of duration $T_{\mathrm{SF}}$ seconds and each SF contains a number frames. The structure of the division of the frame in timeslots (TS) is signalled in the frame composition table (FCT) and all the types of timeslots (i.e., different traffic TS, synchronization TS, etc.) are defined in the timeslot composition table (TCT). See a feasible SF configuration in Fig. 1. Requests generated by the RCSTs depend on the queued traffic at the MAC queues of each terminal and are sent using the standard-defined satellite access control (SAC) messages.

The DVB-RCS standard defines the following three types of capacity request, from highest to lowest priority (we obviate the free capacity assignment (FCA) [18], which may be granted by the NCC, but not requested).

- Constant rate assignment (CRA): the RCST requires a constant rate all the time.

- Rate-based dynamic capacity (RBDC): a bandwidth request (in rate capacity) remains effective until it is updated or timed out. In contrast to CRA, RBDC strategy allows for statistical multiplexing among many RCSTs, resulting in a more efficient use of bandwidth.
- Volume-based dynamic capacity (VBDC): it requires a certain amount of volume capacity to transmit information regardless the way it is done (no constant rate is needed).

The requests generated by all terminals in a beam constitute the inputs of the allocation problem. It is not considered here how terminals generate them. Note that due to the latency of the system (about half a second of RTT), traffic prediction may play an important role. For each bandwidth allocation update, the NCC signals a terminal burst time plan (TBTP) to the RCSTs. It points out which timeslots in the MF-TDMA are assigned to each terminal. With that allocation, the terminal schedules the traffic stored in the MAC queues. It should be noted that the TBTP signals the shape and position of the timeslots in the MF-TDMA, which provides many degrees of freedom for the allocation.

\section{PROPOSED CROSS-LAYER FRAMEWORK}

The DVB-RCS cross-layer framework design can be decomposed into two main parts, which are actually highly coupled, namely: 1) structure imposed in the MF-TDMA and 2) the DBA procedure itself. Note that the performance in the latter depends on the decisions taken in the former. That is, a more structured DBA procedure (with less degrees of freedom) is expected to perform worst. To illustrate the question, imagine that we impose no structure to the transmission and therefore each RCST is allowed to transmit with an arbitrary bandwidth and/or time duration timeslot. This would be in principle a good option. However, the organization of such a collection of TSs with different shape characteristics in the MF-TDMA may be difficult. Indeed, all the possible orderings should be checked and that search over a combinatorial number of possibilities turns the problem into 


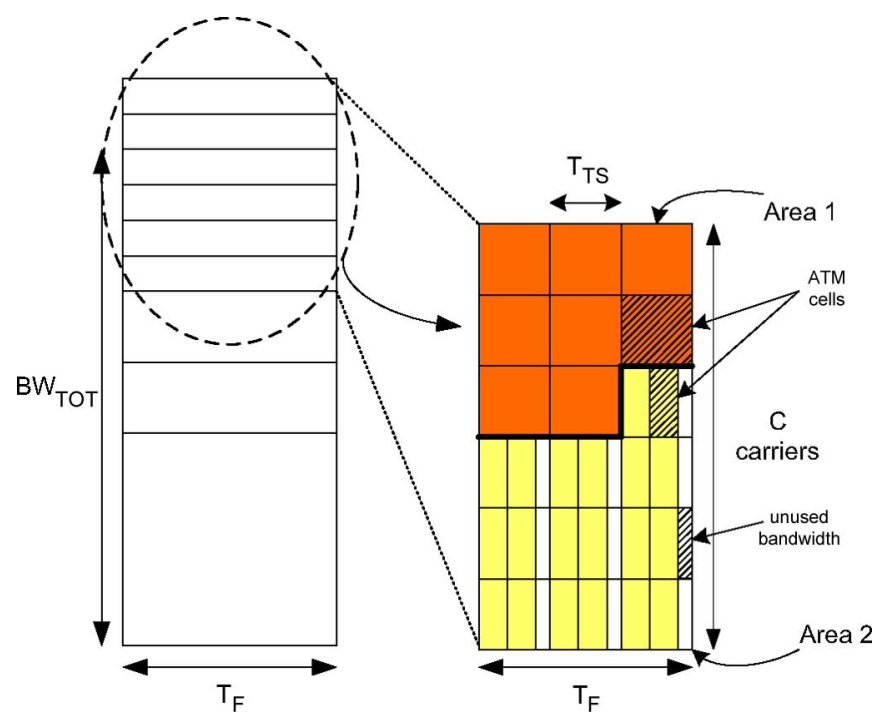

Fig. 2. Scheduling (bandwidth allocation) problem.

NP-hard (not solvable in polynomial time). It is actually meaningful to impose some structure on the MF-TDMA and in this section we deal with those issues, whereas the allocation itself is left to the following section.

In DVB-RCS, the allocation of resources is a reaction to the capacity requests performed by the RCSTs (or users, equivalently). As defined in the standard, the TBTP shall be updated and transmitted every SF, whereas bandwidth is allocated at a frame level. Let us assume that the SF is divided into $N_{F}$ frames using the whole SF bandwidth (BW $\left.\mathrm{BWOT}_{\mathrm{TO}}\right)$ and with a time duration $T_{F}=T_{\mathrm{SF}} / N_{F}$, where $T_{\mathrm{SF}}$ is the $\mathrm{SF}$ duration (typically $265 \mathrm{~ms}$ ). We further assume that $\mathrm{BW}_{\text {TOT }}$ is divided into different carrier types to accommodate different users accounting for different service level agreements (SLAs), terminal equipment, or location, so that an RCST uses only one type of carrier. Under these assumptions, the global allocation is decoupled into $N_{c}$ independent sub-allocations $\left(N_{c}\right.$ standing for the number of carrier types). [See Fig. 2 (left side).]

Thus, the problem we consider consists in multiplexing $N$ users into $C$ carriers of $\mathrm{BW}_{i}$ bandwidth that transmit during $T_{F}$ seconds (see Fig. 2, right side). Without loss of performance and to facilitate upcoming issues, we group all RCSTs that transmit within the same carrier type (equivalently symbol rate) and the same coding rate (in the DVB-RCS, adaptive coding is envisaged to compensate the physical quality of the transmission, i.e., channel conditions). In accordance with ETSI technical specification [19], we refer to each of those groups as an area (we can interpret areas as the earth surface zones where channel conditions are similar).

We consider bandwidth allocation (sometimes referred as scheduling) at MAC layer. In the rest of this paper, we talk about scheduling ATM cells (53 bytes), but the method is valid for any packet length. As an example, the DVB-RCS standard describes moving picture experts group (MPEG) containers (188 bytes) as a possible MAC-layer unit.

We introduce now the key aspect of the proposed framework, which establishes the tradeoff choice between complexity and optimality. A TS of duration $T_{\mathrm{TS}}$ is fixed common to all areas in each allocation process. In Fig. 2, the idea is depicted with

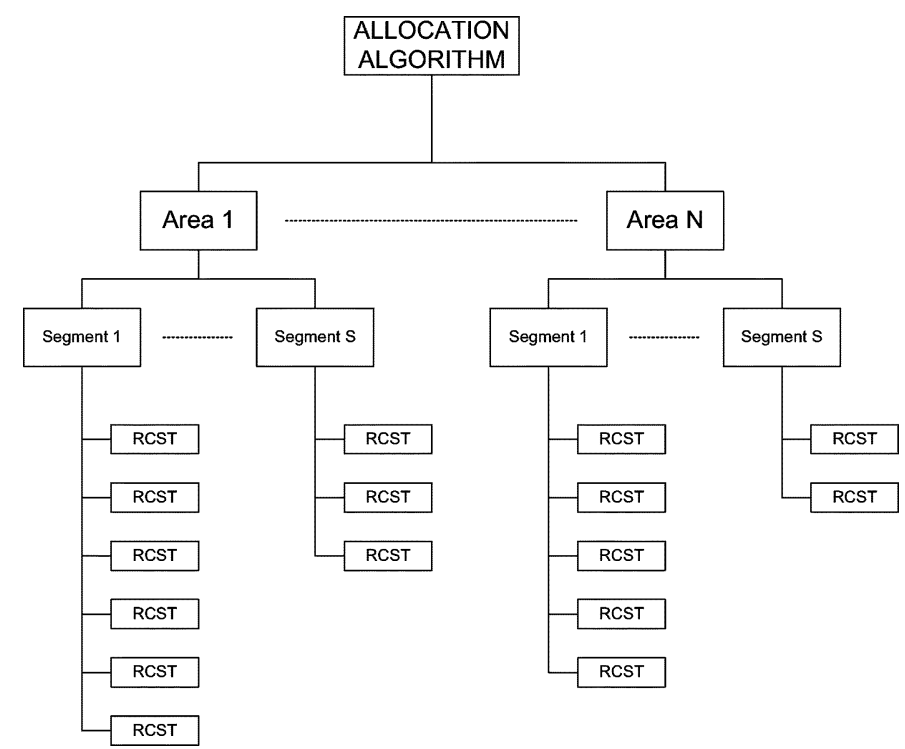

Fig. 3. Bandwidth allocation hierarchy in DVB-RCS.

only two areas. Due to the different coding rates, the transmission time of an ATM cell varies from area to area and so varies the percentage of the time the TS is used, i.e., the bandwidth efficiency per area. Note that it is possible to transmit more than one ATM cell per TS and more specifically, the DVB-RCS takes into account 1, 2, or 4 ATM cells per TS. In this way, cross-layer information from the PHY layer is taken into account. To get cross-layer information from the upper layers, we propose to use the 4 bits available in the field Channel_ID available at SAC messages. Note that this field remains unused if the satellite is transparent. In this way, it is possible to distinguish different traffic types that request capacity using the same type of capacity request. For example, we can consider the QoS defined at IP-level in order to configure a satellite subnetwork as transparent as possible at TCP level.

Further issues of the architecture are both a reduction in signalling and an increased robustness to RCSTs' PHY-layer changes. Regarding signalling, note that TSs with the same characteristics need to be defined only once (with repetitions) in FCT and TCT tables. The reader can find in [17] and [20], different approaches that consider changing the timeslot duration as a function of the coding rate of the area. Advantages and disadvantages of such an approach have already been introduced in this section. Adding now robustness issues to the discussion, note that in full-flexible solutions, the PHY-layer changes in the RCSTs require a whole frame redesign, whereas this is not the case with a common TS approach.

For the sake of completeness, we include the hierarchical bandwidth allocation concept defined in [19]. The motivation is to guarantee some minimum resources to service providers (SPs) as an extra mechanism to grant QoS to their corresponding RCSTs. Note that not necessarily all RCSTs attached to a certain SP see the same channel conditions and thus, they do not belong to the same area in general. It is then adequate to define the segment concept as the group of users that belong to an SP and to guarantee some satellite resources to each segment (e.g., depending on how much they pay). See both area and segment divisions in Fig. 3 and note that they do not define disjoint 
sets, i.e., segment $i$ has some representation in several (if not all) areas. In general, satellite resources must be distributed among areas, segments, and finally among RCSTs.

\section{CROSS-LAYER DYNAMIC BANDWIDTH ALLOCATION ALGORITHMS}

In this section, we develop practical algorithms [17] (i.e., real-time) to compute the DBA in tens of milliseconds (which is small compared to the RTT). Furthermore, the solution is required to make the most efficient use of the available bandwidth and to maximize the system transported capacity at the same time that the fairness among users is maintained. Using known results in game-theory [21], [22], a fair distribution of $P$ resources among $N$ entities responds to the resolution of the following optimization problem, where the objective function is the product [23] of the amount of resources allocated to each entity $x_{i}$. Entity is here a general concept, i.e., it can stand for user, RCST, connection, or whatever

$$
\begin{array}{cl}
\max _{x_{1}, \ldots, x_{N}} & \prod_{i=1}^{N} x_{i}^{p_{i}} \\
\text { s.t. } & \sum_{i=1}^{N} x_{i} \leq P \\
& m_{i} \leq x_{i} \leq d_{i} .
\end{array}
$$

In the previous formulation, $m_{i}$ is the amount of resources guaranteed to entity $i$ and $d_{i}$ stands for its demand. Finally, $p_{i}$ is a weighting factor that represents the importance or priority of that entity (over the whole). It can be proven that the resolution of (1) achieves a proportional fair solution, which is a particular definition of fairness introduced by Kelly et al. [11]. We consider this formulation for DBA in the DVB-RCS, but other applicability examples include scheduling in the DVB-S2 or rate allocation in terrestrial links [24].

Intuitively speaking, a product function forces to share resources just due to the fact that a very low allocation in a single variable $x_{i}$ drops significantly the objective value. Instead, if the sum of $x_{i}$ is considered as the objective function, the obtained solution can be interpreted under the perspective of opportunistic designs: the non-served entity with highest priority reaches its demand or gets all the remaining resources.

The problem in (1) can be easily converted to a convex optimization problem [25] introducing the logarithm in the objective function. The problem is then cast to the network utility maximization (NUM) framework [24]

$$
\begin{array}{cc}
\max _{x_{1}, \ldots, x_{N}} & \sum_{i=1}^{N} p_{i} \cdot \log x_{i} \\
\text { s.t. } & \sum_{i=1}^{N} x_{i} \leq P \\
& m_{i} \leq x_{i} \leq d_{i} .
\end{array}
$$

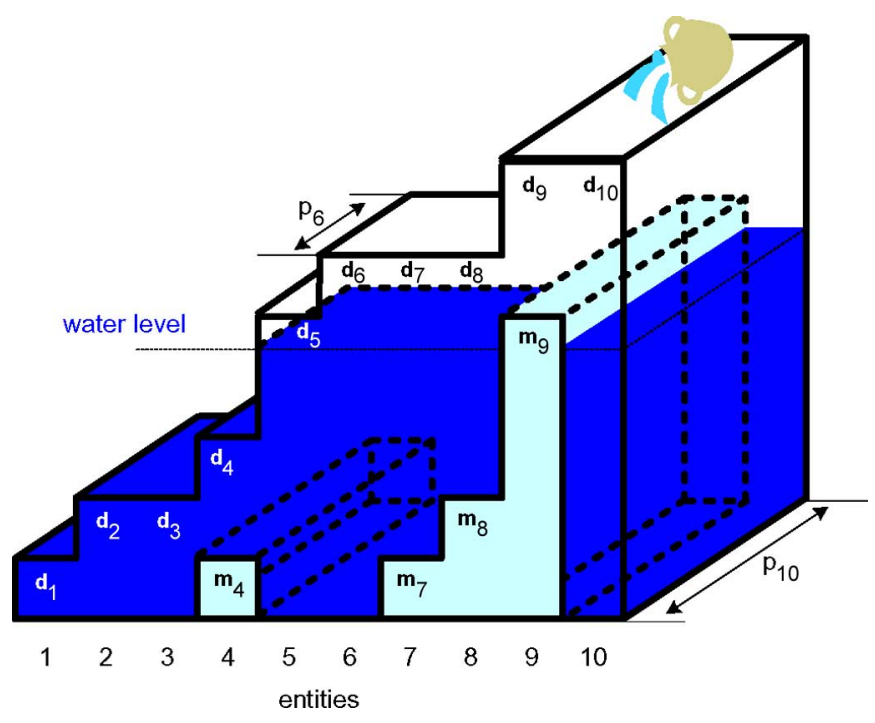

Fig. 4. Fair resource distribution solution.

The utility function per user is, in this case, the logarithm of its allocation. In terms of "utility," the interpretation is that an extra resource is much more useful when the entity has a low number of resources. The resulting problem is analytically solvable using the Karush-Kuhn-Tucker (KKT) conditions [25], which imposes the solution

$$
\left.x_{i}=\frac{p_{i}}{\lambda}\right]_{m_{i}}^{d_{i}} \triangleq \begin{cases}\frac{p_{i}}{\lambda}, & m_{i} \leq \frac{p_{i}}{\lambda} \leq d_{i} \\ m_{i}, & \frac{p_{i}}{\lambda} \leq m_{i} \\ d_{i}, & \frac{p_{i}}{\lambda} \geq d_{i}\end{cases}
$$

where $\lambda$ is a positive value such that $\sum_{i=1}^{N} x_{i}=P$. This value is usually obtained applying the bisection method [26]. However, as the number of entities grows, the method may require excessive computation time [27] (remember that in DVB-RCS the problem has to be solved in tens of milliseconds). Alternatively, the faster the allocation is computed, the highest is the number of users the system can manage.

Graphically, the solution is found by filling a recipient shaped accordingly with the demands, guaranteed resources, and priorities with a quantity $P$ of water [28], as shown in Fig. 4. The solution first assigns the minima ("pale water") and "equally" distributes the remaining water ("strong water").

\section{A. Global DBA Optimization Algorithm}

A slight modification of the optimization problem in (1) allows us to model the DVB-RCS situation

$$
\begin{array}{ll}
\max _{\left\{x_{i, j}\right\}} & \prod_{i, j}\left(x_{i, j} \cdot K_{i}\right)^{p_{i, j}} \\
\text { s.t. } & \sum_{i, j} x_{i, j} \leq P \\
& \left.\quad \frac{m_{i, j}}{K_{i}}\right\rceil \leq x_{i, j} \leq\left\lceil\frac{d_{i, j}}{K_{i}}\right\rceil .
\end{array}
$$




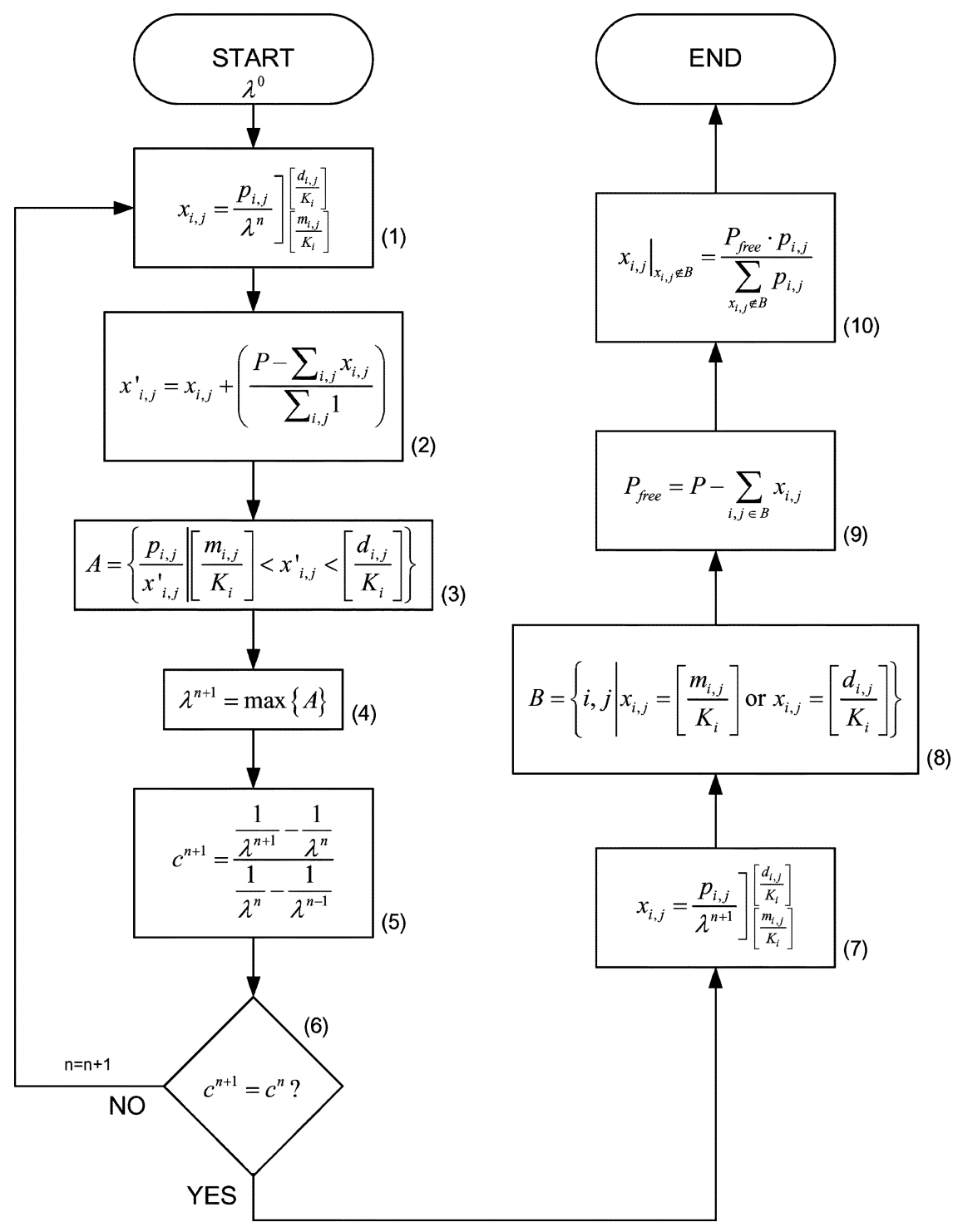

Fig. 5. Joint DBA algorithm.

Now $P$ is the total number of timeslots in the frame, $x_{i, j}$ stands for the amount of timeslots assigned to RCST $i$ with request $j$ and $p_{i, j}$ defines the priority of RCST $i$ with request $j$. Similarly, $d_{i, j}$ and $m_{i, j}$ stand for demands and minimum guaranteed resources (in number of ATM cells). Finally, $K_{i}$ establishes the number of ATM cells that RCST $i$ can transmit in a timeslot (this quantity depends on the time duration of ATM cells and thus on the RCSTs' coding rates) and $\lceil(\cdot)\rceil$ indicates the ceil function. We assume that each SP decides how to distribute the amount of resources that it has been guaranteed with among the RCSTs attached to it, so that $\sum_{i \in S_{k}, j} m_{i, j}=M_{k}$, where $M_{k}$ is the minimum guaranteed resources to the terminals in segment $k, S_{k}$.

Note that we propose to solve a real-valued version of the true problem, i.e., without restricting $x_{i, j}$ to be an integer. The final solution can then be obtained by simple down-rounding and redistribution of the remaining resources to users with highest $p_{i, j}$. Few degradation is expected as the number of ATM cells man- aged by a RCST is assumed to be high. However, other strategies can be adopted, such as variable threshold rounding [29].

We contribute in this section with a novel algorithm to solve (4). It is a good candidate to replace the bisection method due to its computational efficiency and we show this in the results section. The picture of the algorithm can be found in Fig. 5 . Using as an initial guess for $\lambda$, the value $\lambda^{0}=0.5 \cdot[(N /(P-$ $\left.\left.\left.\sum m_{i, j}\right)\right)+\left(1 / \max \left\{d_{i, j}\right\}\right)\right]$, the procedure is as follows:

1) allocate resources using $\lambda^{n}$ and obtaining $x_{i, j}\left(\lambda^{n}\right)$ as in (3);

2) project the previous solution to the capacity limit, $\sum_{i, j} x_{i, j}^{\prime}=P$ and get $x_{i, j}^{\prime}$

3) get a set $A$ of possible new values for $\lambda^{n+1}$ among the users that have a non-saturated solution, $A=\left\{p_{i, j} / x_{i, j}^{\prime} \mid x_{i, j}^{\prime}>\right.$ $\left\lceil m_{i, j} / K_{i}\right\rceil$ or $\left.x_{i, j}^{\prime}<\left\lceil d_{i, j} / K_{i}\right\rceil\right\}$

4) update $\lambda^{n+1}$ with the biggest value in the set;

5) compute the checking value $c^{n+1}=\left(\left(1 /\left(\lambda^{n+1}\right)\right)-\right.$ $\left.\left(1 / \lambda^{n}\right)\right) /\left(\left(1 / \lambda^{n}\right)-\left(1 /\left(\lambda^{n-1}\right)\right)\right)$; 


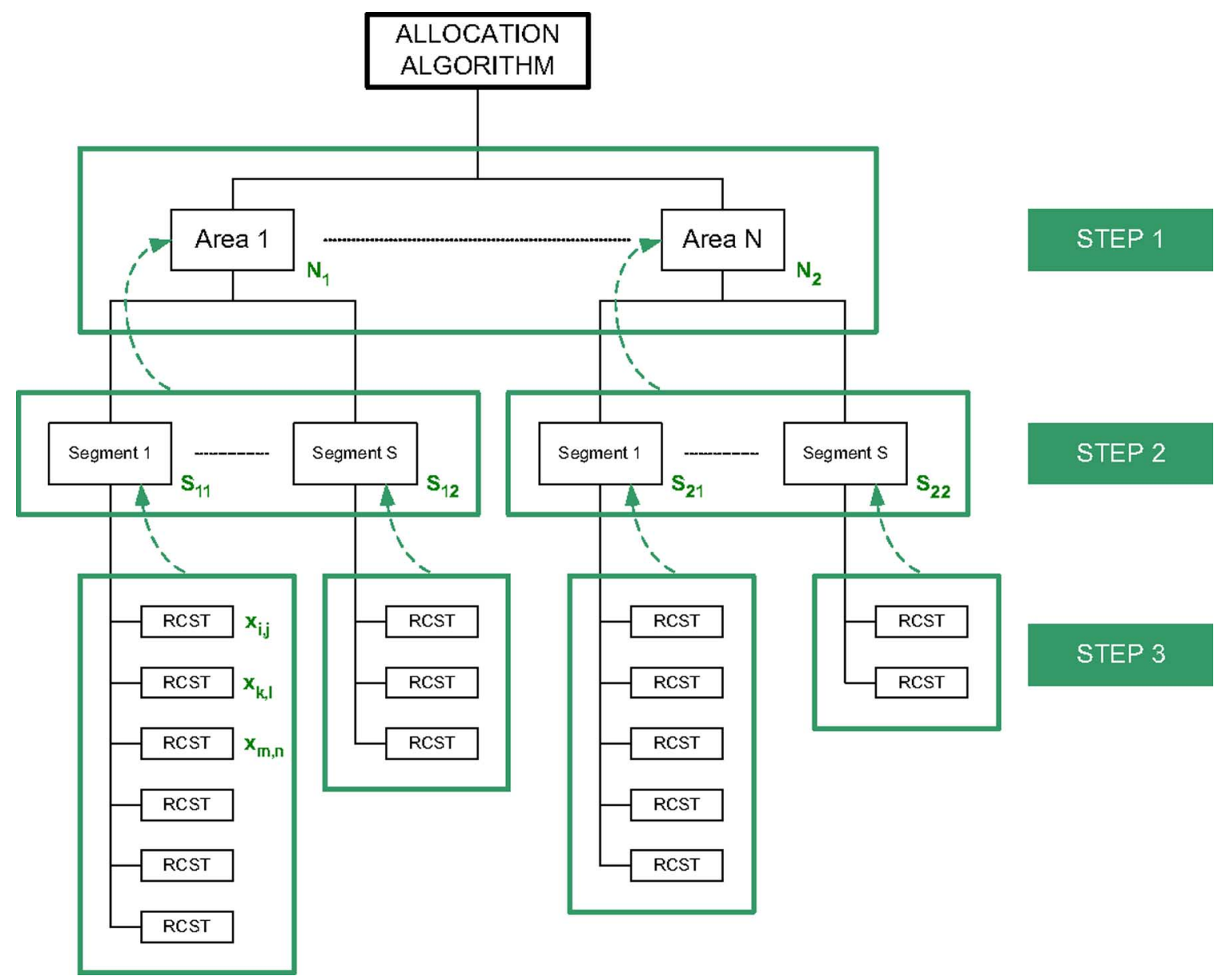

Fig. 6. Hierarchical DBA algorithm.

6) test convergence in $c^{n}$; when $\left|c^{n+1}-c^{n}\right|<\epsilon$, it can be proven that the set $B\left(\lambda^{n+1}\right)=\left\{i, j \mid x_{i, j}\left(\lambda^{n+1}\right)=\right.$ $\left\lceil m_{i, j} / K_{i}\right\rceil$ or $\left.x_{i, j}\left(\lambda^{n+1}\right)=\left\lceil d_{i, j} / K_{i}\right\rceil\right\} \quad$ satisfies $B\left(\lambda^{n+1}\right)=B\left(\lambda^{\infty}\right)$

7) get the set $B\left(\lambda^{n+1}\right)$;

8) calculate the remaining system resources, $P_{\text {free }}$, when resources of users in $B\left(\lambda^{n+1}\right)$ are excluded;

9) finally, distribute $P_{\text {free }}$ depending on priorities and obtain the exact solution as $\left.x_{i, j}\right|_{i, j \notin B}=P_{\text {free }} p_{i, j} / \sum_{i, j \notin B} p_{i, j}$.

Cross-layer information is included in the formulation via two sets of parameters: $\left\{K_{i}\right\}$ and $\left\{p_{i, j}\right\}$. With $K_{i}$ values, we take into account the different coding rates of the RCSTs (PHYlayer information) and thanks to $p_{i, j}$ values, we can prioritize areas, segments, individual RCSTs (depending, for example, on how much they pay), capacity requests, types of traffic, etc. In the most general case, $p_{i, j}$ is a function of MAC-layer parameters (such as type of capacity request) and upper-layer parameters (such as IP-defined QoS).

\section{B. Hierarchical DBA Optimization Algorithm}

Although the previous joint algorithm exhibits good performance results when compared to classical solutions like the bisection method, a suboptimal solution can be used whenever the number of RCSTs makes the problem computationally too hard.
This solution takes profit of the bandwidth allocation hierarchy introduced before. Fig. 6 contains a global representation of the algorithm. The idea is to allocate resources at each level of the frame division hierarchy, namely: areas, segments, and terminals. The allocation at each step is done as before but managing fewer variables.

In the first stage, the overall demands and guaranteed resources per area are computed. These constitute the inputs of the DBA algorithm to obtain the allocation per area. Inside each area, the same procedure is done with segments and again, inside each segment with RCSTs. Priority values $p_{i, j}$ in the last allocation step (step 3 in Fig. 6) can be used for QoS issues. However, they can also be useful in the other steps. For example, we may have the policy to give more resources to areas with higher coding rates as they take more advantage of the satellite link.

\section{Free Capacity Assignment}

Although a heavily loaded system with more traffic demands than system resources is assumed throughout the paper, this is not the only possible situation in systems using DVB-RCS. Indeed, if capacity exceeds the requests, then the problem is to assign the free capacity among the users [18]. The basics of the presented algorithms are still useful in that case with some redefinition of the variables. In that situation, the unused satellite 
capacity is fairly allocated among users by setting $d_{i, j}=\infty$ for all $i, j$.

\section{CRoss-Layer Timeslot Optimization: Joint DBA AND FRAME DESIGN}

A common timeslot design of the frame provides both a simple frame structure and a simple application of efficient DBA algorithms. Still, a proper choice of $T_{\mathrm{TS}}$ is required to maximize system performance.

Let us include this new variable in the previous formulation

$$
\begin{aligned}
\max _{T_{\mathrm{TS},}^{\{}\left\{x_{i, j}\right\}} & \prod_{i, j}\left(x_{i, j} \cdot K\left(T_{\mathrm{TS}}, t_{a(i)}\right)\right)^{p_{i, j}} \\
\text { s.t. } & \sum_{i, j} x_{i, j} \leq P\left(C, T_{F}, T_{\mathrm{TS}}\right) \\
& {\left[\frac{m_{i, j}}{K\left(T_{\mathrm{TS}}, t_{a(i)}\right)}\right\rceil \leq x_{i, j} \leq\left\lceil\frac{d_{i, j}}{K\left(T_{\mathrm{TS}}, t_{a(i)}\right)}\right\rceil } \\
T_{\min } & \leq T_{\mathrm{TS}} \leq T_{\max }
\end{aligned}
$$

where $C$ is the number of carriers, $T_{F}$ the frame duration, and $t_{a(i)}$ is the time duration of an ATM cell transmitted by the $i$ th RCST, where $a(i)$ denotes the area (equivalently coding rate) of RCST $i$.

Developing expressions for $K\left(T_{\mathrm{TS}}, t_{a(i)}\right)=\left\lfloor T_{\mathrm{TS}} / t_{a(i)}\right\rfloor$ and $P\left(C, T_{F}, T_{\mathrm{TS}}\right)=\left\lfloor T_{F} / T_{\mathrm{TS}}\right\rfloor$, the problem can be written as

$$
\begin{aligned}
& \max _{T_{\mathrm{TS}},\left\{x_{i, j}\right\}} \prod_{i, j}\left(x_{i, j} \cdot\left\lfloor\frac{T_{\mathrm{TS}}}{t_{a(i)}}\right\rfloor\right)^{p_{i, j}} \\
& \text { s.t. } \quad \sum_{i, j} x_{i, j} \leq C \cdot\left\lfloor\frac{T_{F}}{T_{\mathrm{TS}}}\right\rfloor \\
& \left\lceil\frac{m_{i, j}}{\left\lfloor\frac{T_{\mathrm{TS}}}{t_{a(i)}}\right\rfloor}\right\rceil \leq x_{i, j} \leq\left\lceil\frac{d_{i, j}}{\left\lfloor\frac{T_{\mathrm{TS}}}{t_{a(i)}}\right\rfloor}\right\rceil, \\
& T_{\min } \leq T_{\mathrm{TS}} \leq T_{\max } \text {. }
\end{aligned}
$$

Clearly, the floor function $(\lfloor\cdot\rfloor)$ converts this joint problem in $T_{\mathrm{TS}}$ and $\left\{x_{i, j}\right\}$ into non-convex. However, it is convex and solved for a fixed value of $T_{\mathrm{TS}}$. The timeslot duration is a continuous variable in the range $\left[T_{\min }, T_{\max }\right]$ but, in this particular problem, not all the values are meaningful. The following lemma considers this issue.

Lemma 1: Starting from a feasible value of $T_{\mathrm{TS}}$ and increasing it, it can only reduce the objective value unless a multiple value of some of the $t_{a(i)}$ 's is reached.

Proof: Start with $T_{\mathrm{TS}}=T_{\min }$ and increase $T_{\mathrm{TS}}$. Stop when a multiple of any of the $t_{a(i)}$ 's is reached. Call this value $T_{\text {mult. }}^{1}$. Then, it is clear that $K_{i}$ 's do not change their value if $T_{\mathrm{TS}} \in\left[T_{\min }, T_{\text {mult }}^{1}\right)$ but $P\left(T_{\text {mult }}^{1}\right) \leq P\left(T_{\text {min }}\right)$. Therefore, the optimal solution of the global problem can not improve until (possibly) $T_{\mathrm{mult}}^{1}$ is reached. The same reasoning is also valid in $T_{\mathrm{TS}} \in\left[T_{\text {mult }}^{1}, T_{\text {mult }}^{2}\right.$ ) (where $T_{\text {mult }}^{2}$ is the next multiple of
TABLE I

AREAS DEFINITION

\begin{tabular}{ccc}
\hline Area identifier & Coding rate & ATM cell duration \\
\hline 1 & $r_{1}=1 / 2$ & $t_{1}=1.06 \mathrm{~ms}$ \\
2 & $r_{2}=2 / 3$ & $t_{2}=0.795 \mathrm{~ms}$ \\
3 & $r_{3}=3 / 4$ & $t_{3}=0.707 \mathrm{~ms}$ \\
4 & $r_{4}=5 / 6$ & $t_{4}=0.636 \mathrm{~ms}$ \\
5 & $r_{5}=7 / 8$ & $t_{5}=0.606 \mathrm{~ms}$ \\
\hline
\end{tabular}

any of the $t_{a(i)}$ 's) and so on. Thus, it holds for the whole range, $T_{\mathrm{TS}} \in\left[T_{\min }, T_{\max }\right]$.

In the DVB-RCS scenario, we can assume few areas and thus few different values of $t_{a(i)}$. Then the list of $T_{\text {mult }}^{k}$ is small and (5) can be efficiently solved via exhaustive (but small) search in $T_{\mathrm{TS}}$ and the usual procedure for $\left\{x_{i, j}\right\}$. The solution for the joint problem (for either global or hierarchical DBA) can be found with the following steps.

- Construct the list of possible values of $T_{\mathrm{TS}}$.

- Reduce the list by suppressing equal values coming from multiples of different $t_{a(i)}$ 's.

- Solve the allocation problem for each of the possible values of $T_{\mathrm{TS}}$.

- Finally, get the $T_{\mathrm{TS}}$ with best objective value in (5).

However, it is not necessary to compute the value of $T_{\mathrm{TS}}$ for each superframe, because 1) $T_{\mathrm{TS}}$ depends on some characteristics of the areas and these are slow time-varying (an area is an aggregation of many RCSTs) and 2) the optimal $T_{\mathrm{TS}}$ is not very sensitive to slight variations of the request as simulation results will show.

\section{RESUlTS AND DISCUSSION}

This section is devoted to show the possibilities of the proposed framework, including operational aspects and performance. First, we define the DVB-RCS scenario under consideration. Then, we evaluate the performance gains obtained with PHY cross-layer information at the NCC. Thereafter, a similar analysis is done for higher-layer information. Finally, we discuss about advantages on computational complexity and signalling overhead.

\section{A. Scenario 1}

Assume a superframe duration of $265 \mathrm{~ms}$, composed of 10 frames $\left(T_{F}=26.5 \mathrm{~ms}\right)$ and consider the sub-allocation problem in 111 carriers of $540 \mathrm{kHz}$ spanning $60 \mathrm{MHz}$ in total.

The PHY layer uses adaptive coding with five possible coding rates, as in the DVB-RCS standard (using convolutional coing). Users are grouped into areas depending on their transmitting rate. See in Table I a description of the areas: associated coding rate and time duration of an ATM cell in the area. Quadrature phase shift keying (QPSK) modulation using a raised cosine pulse with a rolloff factor of 0.35 is assumed. Furthermore, we limit the timeslot duration between $T_{\min }=t_{1}$ and $T_{\max }=3 t_{1}$.

We run Monte Carlo simulations for the joint hierarchical DBA algorithm and timeslot optimization and present results of the first allocation phase (i.e., the resource allocation among 


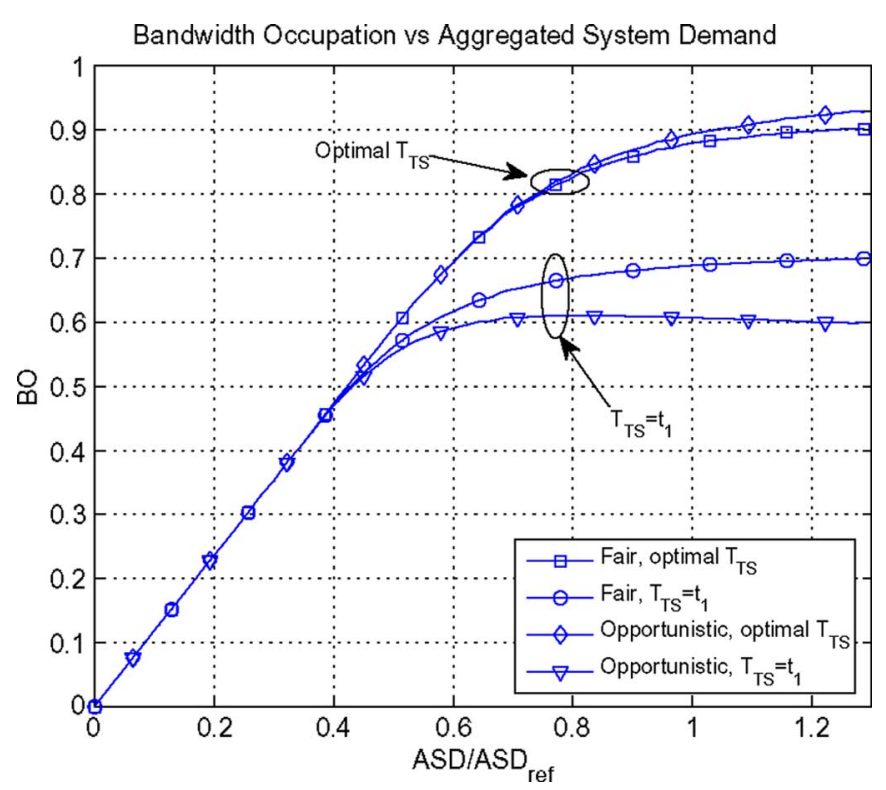

Fig. 7. Bandwidth occupation.

areas). The objective is multiple, namely, to show the performance gains that are obtained through the optimization of the timeslot duration, to focus the advantages of a cross-layer adaptive physical layer, and to depict the mean performance of the system.

Denote the RCSTs aggregated demand as the number of requested ATM cells per area. Define also the aggregated system demand (ASD) as the expected mean of the sum of all demands in all areas. The demand is distributed among the areas using some fixed distribution $\mathcal{V}$. In our case, it represents a realistic scenario that areas with higher rates accumulate more requests as it is expected that most of the RCSTs are in rather good propagation conditions. Note that low rate areas are designed to fulfill the transmission requirements of areas affected by rain. We use the following distribution $\mathcal{V}=[1 / 11,2 / 11,3 / 11,3 / 11,2 / 11]$. Once the ASD is known (it is the statistical mean value), we compute a realization of the demand in each area as $D_{i} \sim$ $\mathcal{U}\left[0,2 \cdot \mathcal{V}_{i} \cdot \mathrm{ASD}\right]$, where $\mathcal{U}[a, b]$ represents a uniform probability density function (pdf) between $a$ and $b$. We further assume the same priority for all RCSTs and no minimum guaranteed resources.

Take as a reference value for the ASD the transported capacity by the system when only the highest rate transmits and the timeslot is exactly fitted, i.e., $T_{\mathrm{TS}}=t_{5}$. This is the maximum amount of information the system can support. In our case, $\mathrm{ASD}_{\text {ref }}=4662 \mathrm{ATM}_{\text {cell }} /$ frame, thus transmitting at $73.82 \mathrm{Mb} / \mathrm{s}$. Note that it may happen that ASD $>\mathrm{ASD}_{\text {ref }}$. Imagine, for example, that the highest rate area asks for $\mathrm{ASD}_{\text {ref }}$. However, this does not imply that other areas ask for no capacity, and thus ASD $>\mathrm{ASD}_{\text {ref }}$ is possible.

Our first analysis in Fig. 7 studies the bandwidth occupation (BO), defined as

$$
\mathrm{BO}=\frac{\sum_{l=1}^{5} N_{l} \cdot K_{l} \cdot t_{l}}{C \cdot T_{F}}
$$

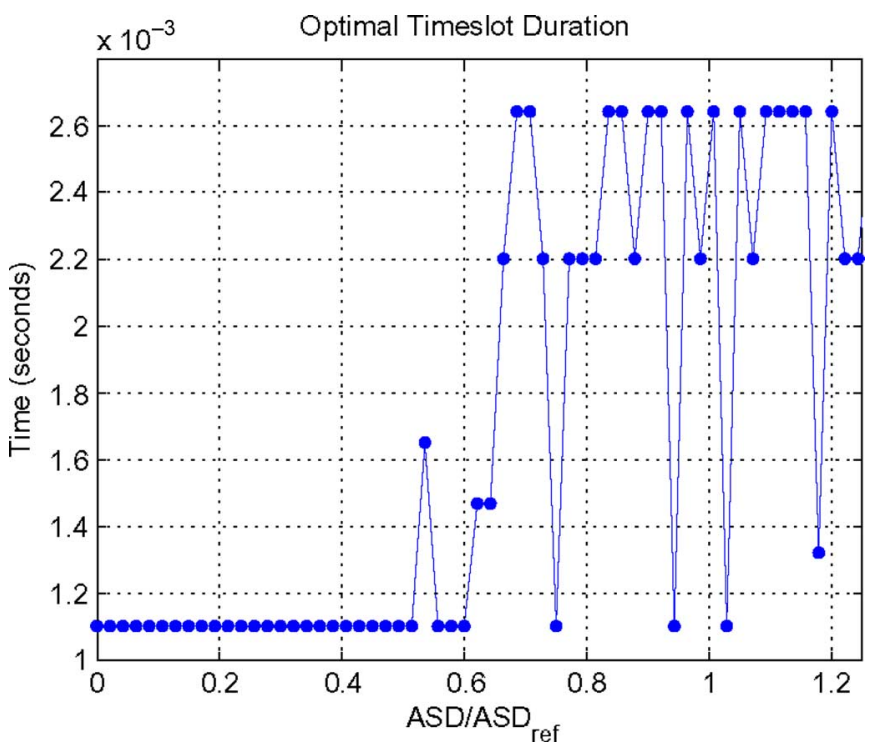

Fig. 8. Optimal timeslot duration.

where $N_{l}$ is the number of timeslots assigned to area $l$. It shows that optimizing $T_{\mathrm{TS}}$ improves significantly the occupation for both fair and opportunistic strategies while it reduces the bandwidth occupation differences between the two designs. In Fig. 8, the reader can find the corresponding optimal values of $T_{\mathrm{TS}}$ for the fair design. Note that slight changes in the system load do not change the election of $T_{\mathrm{TS}}$ (two consecutive $x$-axis points correspond to a 500 ATM cell increase) and therefore a robust design has sense.

Some extra insight is given with the study of the BO values at the relevant $T_{\mathrm{TS}}$ candidates when only one area is requesting. The results can be seen in Table II. Note that some $T_{\mathrm{TS}}$ values exploit better the system occupancy than others depending on which areas we consider active. We have marked the $T_{\mathrm{TS}}=4 t_{4}$ as the configuration that gives better results in the max-min sense (i.e., with no information about demands). If some information is available, for example, if we know that most of the traffic is in areas 3 and 4 , the best choices for $T_{\mathrm{TS}}$ are $T_{\mathrm{TS}}=2 t_{3}$, $T_{\mathrm{TS}}=3 t_{3}$, or $T_{\mathrm{TS}}=4 t_{3}$. This table further justifies that it is not necessary to update $T_{\mathrm{TS}}$ at every superframe.

We also study the normalized transported capacity defined as

$$
T C=\frac{\sum_{l=1}^{5} N_{l} \cdot K_{l}}{\mathrm{ASD}_{\mathrm{ref}}}
$$

Fig. 9 plots the sum of the assigned ATM cells in all areas normalized by $\mathrm{ASD}_{\text {ref }}$. Again, optimizing over $T_{\mathrm{TS}}$ significantly improves the transported capacity (near an $18 \%$ more capacity in the fair case and near a $27 \%$ increase in the opportunistic design). This shows that the increase in BO thanks to $T_{\mathrm{TS}}$ optimization, shown in Fig. 8, effectively translates into an increase in TC. Note that the opportunistic design would reach the maximum possible TC value as ASD increases (independently of the requests distribution), whereas the fair algorithm will generally saturate in a lower value (under 0.8 in this case). This is the price to be paid for being fair. Note also that the difference between the maximum TC's obtained with $T_{\mathrm{TS}}=t_{1}$ and the optimal $T_{\mathrm{TS}}$ establishes the improvement (18\%) achieved thanks 
TABLE II

SYSTEM OCCUPATION ANALYSIS

Bandwidth Occupation

\begin{tabular}{|c|c|c|c|c|c|c|c|c|c|c|c|c|c|}
\hline 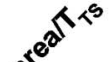 & $t_{1}$ & $2 t_{5}$ & $2 t_{4}$ & $2 t_{3}$ & $2 t_{2}$ & $3 t_{5}$ & $3 t_{4}$ & $3 t_{3}$ & $3 t_{2}$ & $4 t_{5}$ & $4 t_{4}$ & $4 t_{3}$ & $5 t_{5}$ \\
\hline 1 & 1,00 & 0,87 & 0,83 & 0,75 & 0,66 & 0,58 & 0,54 & 1,00 & 0,83 & 0,83 & 0,83 & 0,75 & 0,66 \\
\hline 2 & 0,75 & 0,65 & 0,62 & 0,56 & 1,00 & 0,87 & 0,81 & 0,75 & 0,62 & 0,93 & 0,93 & 0,84 & 0,75 \\
\hline 3 & 0,66 & 0,58 & 0,55 & 1,00 & 0,89 & 0,77 & 0,72 & 1,00 & 0,83 & 0,83 & 0,83 & 1,00 & 0,89 \\
\hline 4 & 0,60 & 0,52 & 1,00 & 0,90 & 0,80 & 0,70 & 0,97 & 0,90 & 0,75 & 0,75 & 1,00 & 0,90 & 0,80 \\
\hline 5 & 0,57 & 1,00 & 0,95 & 0,85 & 0,76 & 1,00 & 0,93 & 0,85 & 0,71 & 0,95 & 0,95 & 0,85 & 0,95 \\
\hline ea & 0,71 & 0,73 & 0,79 & 0,81 & 0,82 & 0,78 & 0,79 & 0,90 & 0,75 & 0,86 &, 91 & 0,87 & 0,81 \\
\hline
\end{tabular}

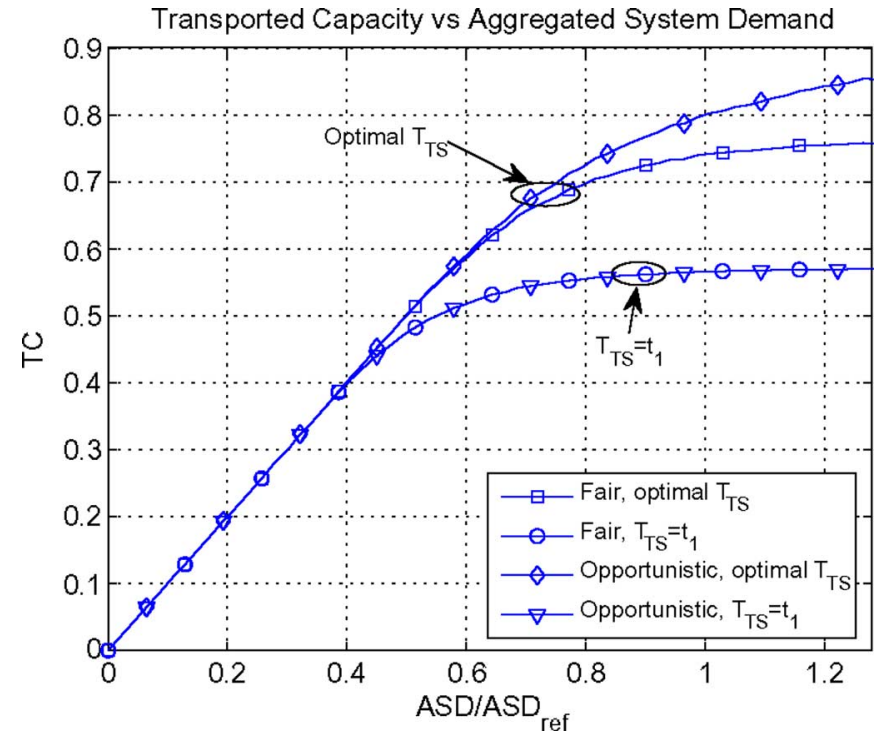

Fig. 9. Transported capacity.

to a cross-layer design of the MAC layer using an adaptive PHY layer.

Fairness differences between the solutions are studied using a fairness index from [30]. Its definition follows: for a given solution $N_{\mathrm{ATM}_{1}}, \ldots, N_{\mathrm{ATM}_{5}}$, we define a new solution set $y_{1}=$ $N_{\mathrm{ATM}_{1}} / N_{\mathrm{ATM}_{1}}^{*}, \ldots, y_{5}=N_{\mathrm{ATM}_{5}} / N_{\mathrm{ATM}_{5}}^{*}$ and we compute the fairness index as

$$
F I=\frac{\left(\sum_{l=1}^{5} y_{l}\right)^{2}}{5 \cdot \sum_{l=1}^{5} y_{l}^{2}}
$$

where $N_{\mathrm{ATM}}^{*}$ is the most "FAIR" solution obtained with the fair algorithm with optimal $T_{\mathrm{TS}}$. Then we compute the $F I$ obtained by the following two solutions:

- the fair solution with $T_{\mathrm{TS}}=t_{1}$;

- the opportunistic solution with optimal $T_{\mathrm{TS}}$, denoted as $T_{\mathrm{TS}}^{\dagger}$, which is obtained applying an opportunistic design (not the fair design as shown before) when compared with the "FAIR" one.

The results are shown in Fig. 10. Note that whereas, 1) exhibits good fairness performance, 2) reduces fairness significantly, which is a reasonable result given that opportunistic solutions are usually unfair or greedy.

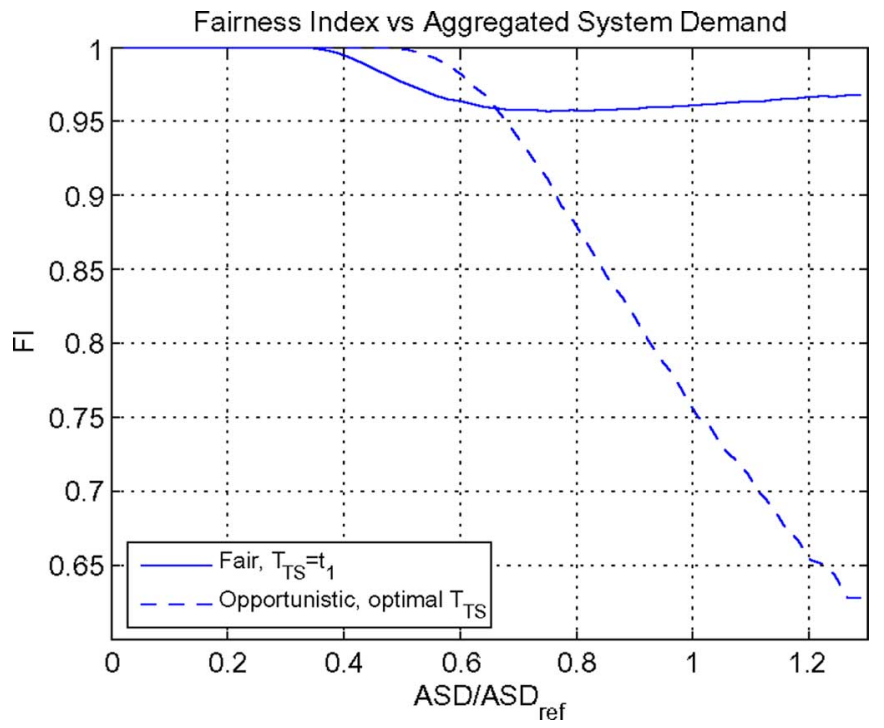

Fig. 10. Fairness study.

\section{B. Scenario 2}

In order to be able to analyze in detail the results of global and hierarchical algorithms, we have considered 24 RCSTs that share 100 timeslots. Results can be extrapolated to larger population and more resources, as we are mainly concerned about how resources are distributed among users. That is, we can scale the problem (in users and resources) and it is still conceptually the same. The scenario is summarized in Tables III and IV. Table III defines each RCST with: 1) assigned area; 2) the number of ATM cells per timeslot; 3) the RCSTs' demands; 4) the RCSTs' minimum guaranteed resources; and 5) the priority of each demand. Table IV defines the segment to which each RCST is attached. In Table V, we have mapped some applications and traffic types to both the priorities in our framework and the types of request in the DVB-RCS.

We plot in Fig. 11 the result of applying the global DBA algorithm. In black tone there is the final allocation, in pale gray tone the minimum guaranteed resources, and in dark grey tone the demands for the 24 RCSTs. The real-valued solution is simply rounded down to adapt it to the DVB-RCS characteristics. Note that RCSTs with higher priority are allocated a higher number of resources than RCSTs with lower priority, even when the request type is the same (VBDC). This issue is captured by the dotted horizontal lines in the figure. For example, we can 
TABLE III

RCSTS DEFINITION

\begin{tabular}{cccccc}
\hline Area identifier & RCST & ATM cells per TS & Requests & Minimums & Priorities \\
\hline 1 & $1-2$ & 1 & {$[15,16]$} & {$[2,0]$} & {$[1.75,1.25]$} \\
2 & $3-6$ & 1 & {$[9,19,14,5]$} & {$[0,1,2,0]$} & {$[1.5,2,1.25,1.75]$} \\
3 & $7-13$ & 2 & {$[17,13,4,5,13,13,8]$} & {$[1,2,2,2,0,1,1]$} & {$[1.75,2,1.5,2,1.25,1.5,1.25]$} \\
4 & $14-20$ & 2 & {$[12,10,2,2,7,1,8]$} & {$[1,0,2,2,2,1,2]$} & {$[1.5,1.5,1.75,1.75,2,1.25,1]$} \\
5 & $21-24$ & 2 & {$[14,3,2,13]$} & {$[0,1,2,3]$} & {$[1.5,2,1,1.75]$} \\
\hline
\end{tabular}

TABLE IV

ASSIGNMENT OF RCSTS TO SEGMENTS

\begin{tabular}{cc}
\hline Segment identifier & Attached RCSTs \\
\hline 1 & {$[1,3,4,7,8,9,14,15,16,17,21,22]$} \\
2 & {$[2,5,6,10,11,12,13,18,19,20,23,24]$} \\
\hline
\end{tabular}

TABLE V

TYPES OF REQUEST

\begin{tabular}{lcc}
\hline Requested QoS & Associated priorities & Request type \\
\hline VoIP & 2 & RBDC \\
Video streaming & 1.75 & RBDC \\
Telnet, gaming & 1.5 & VBDC \\
Web browsing & 1.25 & VBDC \\
FTP, SMTP & 1 & VBDC \\
\hline
\end{tabular}

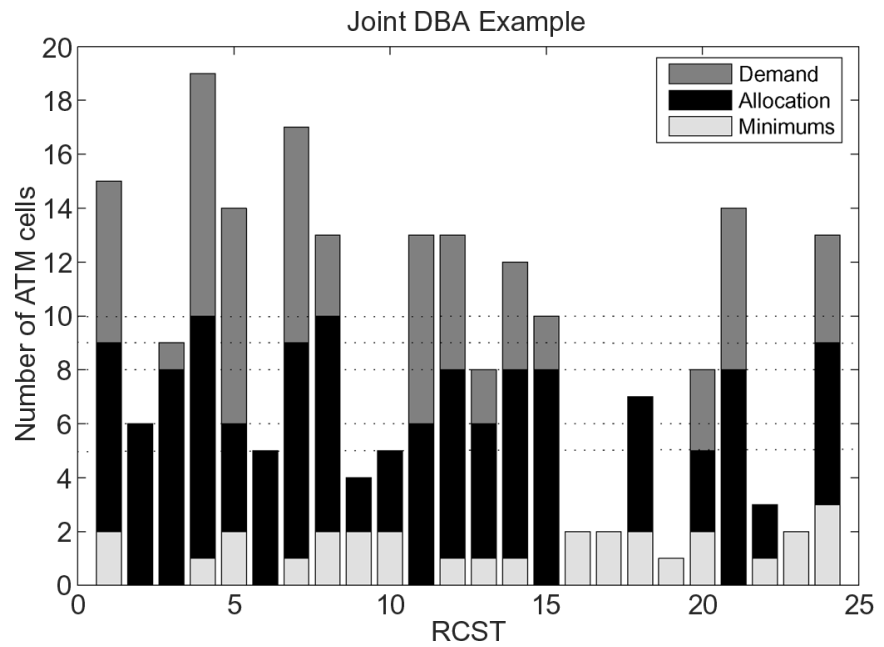

Fig. 11. Joint DBA example.

compare RCSTs 12 and 11; the former receives a larger allocation thanks to its larger priority despite they have requested the same amount of resources. RCST 12 is streaming video whereas RCST 11 is simply web browsing.

We compare now the global and hierarchical DBA algorithms using the same scenario. The final allocation of both designs can be seen in Fig. 12: in pale gray tone, the allocation computed by the hierarchical DBA algorithm; both in dark tone and black stars, the allocation with the global DBA algorithm. Note with this simple example the difference between them and thus, the sub-optimality of the hierarchical approach. To exemplify

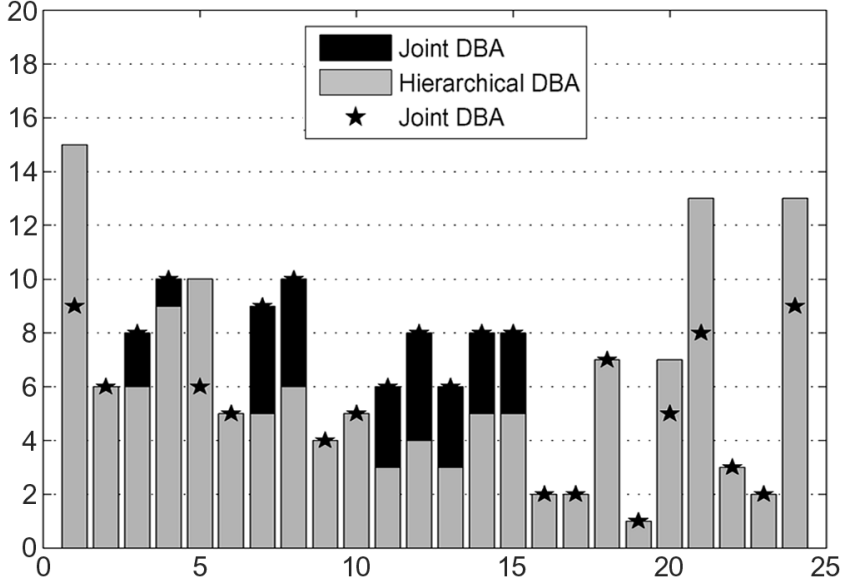

Fig. 12. Hierarchical versus joint DBA.

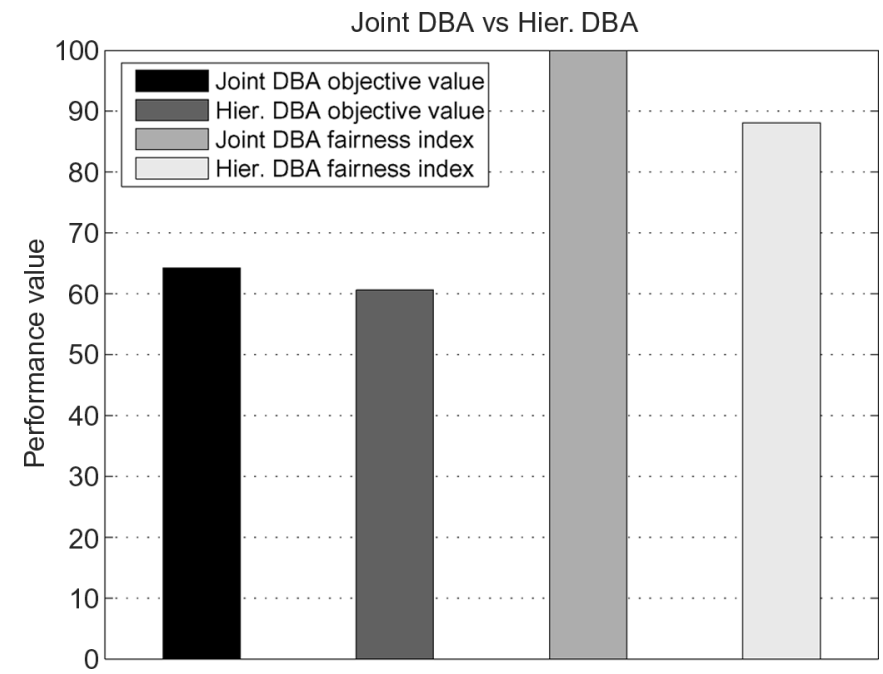

Fig. 13. DBA algorithms performance.

the different performance, we have plotted in Fig. 13 both the logarithm of the objective value in (4) and the fairness index as previously defined (the global solution is considered the optimal one and thus $100 \%$ fair). Fairness is significantly reduced $(12 \%)$ and the values of the objective function are significantly different (they are in logarithmic scale).

Finally, Table VI compares the amount of traffic in the number of ATM cells dedicated to each application (as defined in Table V) both considering priorities and without taking them into account. Note that priorities effectively balance the traffic 
TABLE VI

Traffic (IN Number of ATM CELlS) With/Without Priorities

\begin{tabular}{cccc}
\hline Traffic type & With priorities & Without priorities & $\begin{array}{c}\text { Opportunistic } \\
\text { allocation }\end{array}$ \\
\hline VoIP & 35 & 31 & 47 \\
Video streaming & 36 & 33 & 54 \\
Telnet, gaming & 44 & 44 & 48 \\
Web browsing & 25 & 31 & 0 \\
FTP, SMTP & 7 & 10 & 0 \\
\hline
\end{tabular}

towards the most stringent applications, proving the interest of the proposed cross-layer allocation algorithm.

\section{Computational Complexity and Signalling}

Last, but not least, we discuss over computation time and signalling performance. It is indeed an extremely important issue in the DBA of the DVB-RCS and the effort is required to develop time-efficient algorithms, capable of computing the resource allocation of maybe thousands of RCST requests in a superframe time. In this subsection, we compute both the global DBA algorithm and the bisection method applied to (1) in a Pentium-mobile processor running at $1.73 \mathrm{GHz}$. The inputs of the algorithm are discrete (integer) uniform random variables with different thresholds: 1) $d_{i} \sim \mathcal{U}[1,20]$; 2) $m_{i} \sim \mathcal{U}[0,3]$; and 3) $K_{i} \sim \mathcal{U}[1,2]$. Priorities are also discrete, $p_{i} \sim \mathcal{U}[1,2]$, but with a step of 0.25 (i.e., $p_{i} \in\{1,1.25,1.5,1.75,2\}$ ). See in Fig. 14 the computational time of both algorithms as the number of RCSTs grows.

Note that the global DBA algorithm solves the allocation in approximately half the time the bisection method requires. In the DVB-RCS, within the $100 \mathrm{~ms}$ available time, the bisection method can manage around 8500 users while the global DBA algorithm could cope with around 18000 . Furthermore, we have only required unit precision to the bisection method in the quantity $\left|\sum_{i, j} x_{i, j}-P\right|$ (as the solution is rounded in the DVB-RCS case) while the proposed global DBA algorithm finds the exact solution. Note also that computational time grows more linearly in the proposed algorithm, which is a desirable feature.

Finally, we discuss signalling issues using Scenario 1. We consider now two approaches: 1) common timeslots for all areas and 2) different timeslot duration for each area. We consider here the signalling required in the FCT. There are not significant differences in the TCT as the five types of timeslots have to be described in the TCT for both approaches (i.e., the timeslots have different coding rates). The flexible approach requires to signal every timeslot in the frame as no structure is known a priori. Transmitting only through the highest rate, it is possible to place 4662 timeslots and 2664 are possible if only the lowest rate transmits. Assuming the mean value between these (i.e., 3663), the FCT transmits $(174+3663.72)$ bits in $26.5 \mathrm{~ms}$, resulting in a signalling rate of about $9.5 \mathrm{Mb} / \mathrm{s}$. On the other hand, our approach only needs to signal the timeslot at the beginning of the carrier and indicate the number of repetitions. This turns into $(174+111 \cdot 72)$ bits and about $300 \mathrm{~kb} / \mathrm{s}$ of signalling rate.

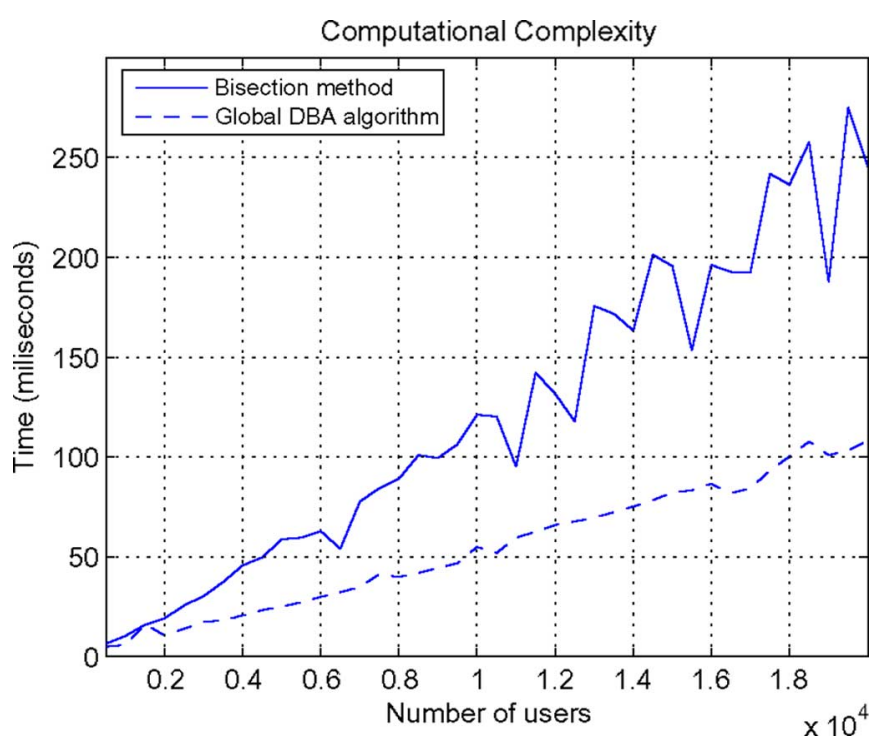

Fig. 14. Computational time (joint DBA and bisection method).

\section{CONCLUSION}

The contributions of this paper are an optimized framework for the DVB-RCS along with fair and time-efficient DBA algorithms that take into account cross-layer information both from the lower layer (PHY layer) and the upper layers (IP/APP-layers in our example).

The proposed approach is compliant both with the DVB-RCS standard and with the ETSI technical specifications. Unlike other approaches, our contribution fixes some structure, the timeslot, common to all areas (i.e., coding rates). This results in reduced signalling, increased robustness to PHY-layer changes, and reduced complexity of the subsequent resource allocation. Then, depending on the spectral efficiency of the RCSTs within a given area, one or more ATM cells can be transmitted. The timeslot is optimized either for each allocation cycle or in a max-min sense. Results show that a good usage of the satellite bandwidth is achieved with this strategy and that the MAC cross-layer design enabled by an adaptive PHY layer reports significant gains.

Thereafter, time-efficient algorithms have been presented for the allocation of bandwidths to RCSTs (in global and hierarchical approaches). The basic algorithm is able to find the exact solution to the problem with less than half the time of the widely used bisection method. We have exemplified both algorithms, showing the sub-optimality of the hierarchical approach. This makes it even more important to solve the global allocation with little complexity.

Finally, the use of priorities at MAC-layer gives continuity to the QoS requirements defined at upper layers, such as IP-layer or APP-layer. Priorities can be explicitly signalled to the NCC or alternatively, the NCC can extract this information from the traffic.

\section{REFERENCES}

[1] T. Le-Ngoc and T. Elshabrawy, "Broadband satellite access for interactive multimedia services," Space Commun., vol. 17, pp. 35-48, 2001. 
[2] ETSI, Sophia-Antipolis, France, "Digital video broadcasting (DVB); framing structure, channel coding and modulation for $11 / 12 \mathrm{GHz}$ satellite services," ETSI EN 300 421, Aug. 1997.

[3] ETSI, Sophia-Antipolis, France, "Digital video broadcasting (DVB); second generation framing structure, channel coding and modulation systems for broadcasting, interactive services, news gathering and other broadband satellite applications," ETSI EN 302 307, Mar. 2005.

[4] ETSI, Sophia-Antipolis, France, "Digital video broadcasting (DVB); Interaction channel for satellite distribution systems," ETSI EN 301 790, Apr. 2005.

[5] ETSI, Sophia-Antipolis, France, "Digital video broadcasting (DVB); Interaction channel for satellite distribution systems; Guidelines for the use of EN 301 790," ETSI TR 101 790, Jan. 2003.

[6] R. Rinaldo, M. A. Vázquez-Castro, and A. Morello, "DVBS2 ACM modes for IP and MPEG unicast applications," Int. J. Sat. Commun., vol. 22 , no. 3, pp. 367-399, May/Jun. 2004

[7] G. Albertazzi, S. Cioni, G. E. Corazza, M. Neri, R. Pedone, P. Salmi, A. Vanelli-Coralli, and M. Villanti, "On the adaptive DVB-S2 physical layer: Design and performance," IEEE Trans. Wireless Commun., vol. 12, no. 6, pp. 62-68, Dec. 2005.

[8] L. Chisci, R. Fantacci, F. Francioli, and T. Pecorella, "Dynamic bandwidth allocation via distributed predictive control in satellite networks," in Proc. 1st Int. Symp. Control, Commun. Signal Process., 2004, pp. 373-376.

[9] A. Gotta, F. Portoti, and R. Sechhi, "Simulating dynamic bandwidth allocation on satellite links," presented at the ACM Int. Conf. Series, Pisa, Italy, 2006.

[10] H. Skinnemoen, A. Vermesan, A. Iuoras, G. Adams, and X. Lobao, "VoIP over DVB-RCS with QoS and bandwidth on demand," IEEE Trans. Wireless Commun., vol. 12, no. 5, pp. 46-53, Oct. 2005.

[11] F. P. Kelly, A. Maulloo, and D. Tan, "Rate control for communication networks: Shadow prices, proportional fairness and stability," J. Oper. Res. Soc., vol. 49, no. 3, pp. 237-252, Mar. 1998.

[12] R. Srikant, The Mathematics of Internet Congestion Control. Cambridge, MA: Birkhäuser, 2004.

[13] G. Açar and C. Rosenberg, "Algorithms to compute for bandwidth on demand requests in a satellite access unit," presented at the 5th Ka Band Utilization Conf., Taormina, Italy, 1999.

[14] G. Açar and C. Rosenberg, "Weighted fair bandwidth-on-demand (WFBoD) for geostationary satellite networks with on-board processing," Comput. Netw., vol. 39, no. 1, pp. 5-20, May 2002.

[15] F. D. Priscoli and A. Pietrabissa, "Design of a bandwidth-on-demand (BoD) protocol for satellite networks modelled as time-delay systems," Automatica, vol. 40, no. 5, pp. 729-741, May 2004.

[16] N. Iuoras and L.-N. Tho, "Dynamic capacity allocation for quality-ofservice support in IP-based satellite networks," IEEE Trans. Wireless Commun., vol. 12, no. 5, pp. 14-20, Oct. 2005.

[17] K.-D. Lee and K.-N. Chang, "A real-time algorithm for timeslot assignment in multirate return channels of interactive satellite multimedia networks," IEEE J. Sel. Areas Commun., vol. 22, no. 3, pp. 518-528, Apr. 2004

[18] J. Neale and A. K. Mohsen, "Impact of CF-DAMA on TCP via satellite performance," in Proc. Global Telecommun. Conf. (GLOBECOM), Nov. 2001, vol. 4, pp. 2687-2691.

[19] ETSI, Sophia-Antipolis, France, "Satellite Earth stations and systems (SES); Broadband satellite multimedia (BSM) services and architectures: QoS functional architecture," ETSI TS 102 462, Dec. 2005.

[20] M. A. Vázquez Castro, M. Ruggiano, L. S. Ronga, and M. Werner, "Uplink capacity limits for DVB-RCS systems with dynamic framing and adaptive coding," presented at the 23rd Int. Commun. Sat. Syst. Conf., Rome, Italy, Sep. 2005.

[21] A. Muthoo, Bargaining Theory With Applications. Cambridge, U.K.: Cambridge Univ. Press, 1999.

[22] H. Yaiche, R. R. Mazumdar, and C. Rosenberg, "A game theoretic framework for bandwidth allocation and pricing in broadband networks," IEEE/ACM Trans. Netw., vol. 8, no. 5, pp. 667-678, Oct. 2000.

[23] R. Mazumdar, L. G. Mason, and C. Douligeris, "Fairness in network optimal flow control: Optimality of product forms," IEEE Trans. Commun., vol. 39, no. 5, pp. 775-782, May 1991.

[24] D. P. Palomar and M. Chiang, "Alternative decompositions for distributed maximization of network utility: Framework and applications," presented at the IEEE INFOCOM, Barcelona, Spain, Apr. 2006.
[25] L. Boyd and S. Vandenberghe, Convex Optimization. Cambridge, U.K.: Cambridge Univ. Press, 2003.

[26] G. V. Reklaitis, A. Ravindran, and K. M. Ragsdell, Engineering Optimization: Methods and Applications. New York: Wiley-Interscience, 1983.

[27] A. Girard, C. Rosenberg, and M. Khemiri, "Networking 2000," in Fairness and aggregation: A primal decomposition study. New York: Springer-Verlag, May 2000, pp. 667-678.

[28] D. P. Palomar and J. Fonollosa, "Practical algorithms for a family of waterfilling solutions," IEEE Trans. Signal Process., vol. 53, no. 2, pp. 686-695, Feb. 2005.

[29] L. Xiao, M. Johansson, H. Hindi, S. Boyd, and A. Goldsmith, "Joint optimization of communication rates and linear systems," IEEE Trans. Autom. Control, vol. 48, no. 1, pp. 148-153, Jan. 2003.

[30] R. Jain, D. Chiu, and W. Hawe, "A quantitative measure of fairness and discrimination for resource allocation in shared systems," Digital Equipment Corp., Maynard, MA, Tech. Rep. DEC TR-301, Sep. 1984.

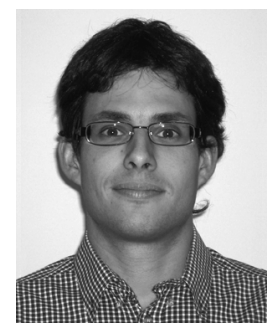

Antoni Morell (S'02) received the electrical engineering degree and the Diploma of Advanced Studies from the Polytechnic University of Catalonia (UPC), Barcelona, Spain, in 2002 (DEA) and 2005, respectively.

In September 2005, he joined the Department of Telecommunications and System Engineering, Universitat Autònoma de Barcelona (UAB), Barcelona, Spain. From April 2002 to September 2005, he was a Research Assistant with UPC, where he enjoyed a predoctoral grant from the Spanish government. His research interests include cross-layer optimization, convex optimization and decomposition techniques, and network utility maximization.

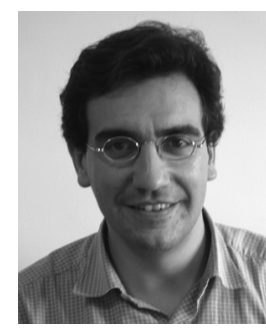

Gonzalo Seco-Granados (M'02) received the Ph.D degree in telecommunication engineering from Universitat Politècnica de Catalunya, Barcelona, Spain, in 2000, and the M.B.A. degree from IESE-University of Navarra, Barcelona, Spain, in 2002.

Since 2006, he has been an Associate Professor with the Department of Telecommunications and Systems Engineering, Universitat Autònoma de Barcelona, Barcelona, Spain. Until 2005, he was member of the technical staff with the Electrical Engineering Department, European Space Research and Technology Center (ESTEC), European Space Agency, Noordwijk, The Netherlands, where he was involved in the Galileo Project. He led the activities on indoor positioning. His research interests include signal processing and resource allocation for ad hoc networks and location-based communications.

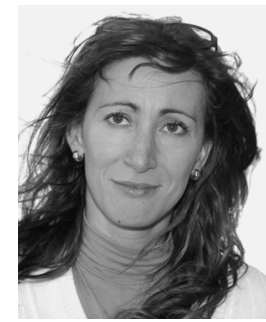

María Ángeles Vázquez-Castro (M’99) received the Telecommunication Engineer degree and the $\mathrm{Ph} . \mathrm{D}$. degree (cum laude) from the Polytechnic University of Vigo, Vigo, Spain, in 1994 and 1998, respectively.

She is currently an Associate Professor with the Universitat Autònoma de Barcelona, Barcelona, Spain. During 2002-2004, she was a Research Fellow with the European Space Agency and a Visiting Researcher with the University of Southern California, Los Angeles, in 2000. She leads a research group on wireless communications that belongs to the European Network of Excellence on Satellite Communications that has been awarded as "Emerging Research Group" from the Catalonian Government. She has lead and participated in several national and international research projects and has co-authorised a number of books and papers, holds one patent and contributes to the standardization bodies ITU, DVB, and ETSI. Her current research interests include cross-layer and cooperative design of wireless terrestrial and satellite systems. 